\title{
Les rapports entre médecine et chirurgie dans la grande école anglaise de William et John Hunter*
}

\section{Par Othmar Keel}

Comme on le sait, le rapprochement entre la médecine et la chirurgie (et l'adoption par la médecine du point de vue localiste de la chirurgie) qui s'est produit au $18^{\mathrm{e}}$ siècle et au début $\mathrm{du} 19^{\mathrm{e}}$ siècle a été un facteur très important de la constitution d'une conception anatomo-clinique de la maladie. Plusieurs auteurs ont insisté sur le fait que les chirurgiens français, et notamment parisiens, du $18^{\mathrm{e}}$ siècle avaient joué un rôle de premier plan dans la préparation de la naissance de la médecine anatomo-clinique. Selon ces auteurs, la clinique chirurgicale du $18^{\mathrm{e}}$ siècle en France a précédé la clinique médicale qui n'est apparue qu'au $19^{\mathrm{e}}$ siècle et elle lui a servi de modèle à la fois sur le plan institutionnel et sur le plan théorique ${ }^{1}$. Au $18^{\mathrm{e}}$ siècle, a-t-on dit, les chirurgiens français avaient déjà l'at titude de la médecine anatomo-clinique et le diagnostic chirurgical était, par sa nature même, facteur de progrès, car il localisait la maladie ${ }^{2}$.

Il est vrai, sans doute, que la clinique chirurgicale a joué en France, et notamment, à Paris, un rôle important pour stimuler une orientation anatomique de la clinique médicale. Cependant, $O$. Temkin avait déjà souligné, il y a longtemps, que les chirurgiens étrangers, et notamment anglais, du $18^{\mathrm{e}}$ siècle, avaient aussi une approche localiste et anatomostructurale de la maladie ${ }^{3}$. Bien avant Morgagni, explique Temkin, les chirurgiens faisaient des diagnostics en mettant en corrélation le tableau clinique et des modifications de structure dans les organes. Autrement dit, ils faisaient exactement ce que les écoles de Paris et de Vienne allaient faire pour les maladies internes. Les chirurgiens ne pouvaient pas opérer sans avoir visualisé d'abord l'altération anatomique qui était à l'origine de la maladie. Tout ceci, selon Temkin, ressort tout autant des écrits des chirurgiens de l'école anglaise de Cheselden et de Pott, que de ceux de l'école française comme Jean-Louis Petit. C'est d'ailleurs dans la plupart des pays d'Europe que, au $18^{\mathrm{e}}$ siècle, l'orientation anatomique de la médecine a été stimulée, au

\footnotetext{
* Les recherches pour cette étude ont été rendues possibles par une subvention du Conseil de Recherches en Sciences Humaines du Canada. Nous remercions M.P. Keating pour son assistance au cours de ces recherches.
} 
moins en partie, par une convergence croissante entre la médecine et la chirurgie, les deux disciplines cultivant l'anatomie pathologique ${ }^{4}$. Et un peu partout en Europe, les médecins ont commencé à valoriser et à adopter une attitude manuelle et expérimentale dans leur pratique analogue à celle qui était déjà au centre de la chirurgie ${ }^{5}$.

Au début du $19^{\mathrm{e}}$ siècle, selon Temkin, cette importance que prend la chirurgie dans le domaine de l'art de guérir est encore plus évidente en Angleterre qu'en France. Les figures médicales principales en Angleterre entre 1800 et 1830, explique-t-il, étaient les chirurgiens Abernethy, W. Lawrence, Sir A. Cooper, E. Home, B. Brodie, C. Bell. Même plusieurs des médecins anglais les plus remarquables au cours de cette période avaient été sous la tutelle des chirurgiens: M. Baillie était un neveu et un disciple des Hunters: son travail en anatomie pathologique était largement basé sur la collection de W. Hunter. Jenner avait été un élève de John Hunter, et ce n'est qu'en 1792, ayant acquis un diplôme médical de l'Université Saint Andrew's, qu'il avait abandonné le côté chirurgical de sa pratique. R. Bright devait à A. Cooper, sous la direction duquel il avait travaillé à l'hôpital Guy en 1811, son goût pour l'anatomie pathologique ${ }^{6}$. Il faut souligner ici que toutes ces grandes figures médicales anglaises de la fin du $18^{\mathrm{e}}$ et du début du $19^{\mathrm{e}}$ siècles mentionnées par Temkin sont des élèves des Hunters ou de l'école huntérienne. Cette école a joué un rôle de premier plan dans le rapprochement de la chirurgie et de la médecine en Angleterre. Elle a également eu une fonction décisive, en Angleterre et dans d'autres pays, dans la stimulation d'une approche localiste et structurale (ou anatomo-clinique) de la maladie en médecine. Cette orientation existait déjà en médecine en Angleterre, comme on le voit par l'enseignement et les travaux de médecins et d'anatomistes comme J.Douglas et F. Nicholls, mais elle était encore limitée? ${ }^{7}$ L'école huntérienne lui a donné une impulsion déterminante. Temkin a dit que pour démontrer que les chirurgiens avaient de l'influence sur la médecine à la fin du $18^{\mathrm{e}}$ siècle, il suffirait de citer le nom de John Hunter ${ }^{8}$. Par ailleurs, Charles Bell avait écrit en 1812 "The school founded by the Hunters has made all the anatomists of the present day at home and abroad". ${ }^{9}$ Mais la pathologie médicale occupe, comme nous le verrons, une place essentielle dans la formation et dans l'œuvre des Hunters, de sorte qu'il y a influence réciproque de la chirurgie et de la médecine l'une sur l'autre et non seulement influence de la chirurgie sur la médecine.

John Hunter (1728-1793) est généralement reconnu en histoire de la médecine pour avoir mis en avant une théorie physiologique de la maladie 
qui définit celle-ci comme dysfonction ou comme une modification pathologique des actions normales (saines) des organes et des parties. Ces modifications entraînent des lésions des organes et des tissus qu'il faut apprendre à connaître par la dissection anatomo-pathologique ${ }^{10}$. En cela, Hunter a opéré une rupture radicale avec les conceptions noxotaxiques et essentialistes traditionnelles de la maladie ${ }^{11}$. Les contemporains et les historiens ont reconnu à Hunter le mérite d'avoir changé le visage de la médecine du $18^{\mathrm{e}}$ siècle en Grande-Bretagne en développant la recherche dans toutes ses branches ${ }^{12}$. On considère que Hunter a rendu scientifique non seulement la chirurgie mais aussi la médecine et qu'il a jeté les bases de l'anatomie et de la physiologie comparées en Grande-Bretagne. Comme l'a dit George Mather, le grand but de la vie de John Hunter a été d'étudier et d'enseigner la physiologie comme la seule vraie base de la chirurgie et de la médecine ${ }^{13}$. Le huntérien William Lawrence avait déjà écrit, en 1819, (leçons données au Royal college of Surgeons): "Thus we find that anatomy, physiology, morbid anatomy and pathology are mutually related and intimately connected. Although called separate Sciences, they are in truth, parts of one system ... The collection of this college was formed and is now arranged in conformity to the views just alluded to... Mr. Hunter was the first in this country who investigated disease in a strictly physiological method ...". ${ }^{14}$

Comment expliquer que John Hunter, qui d'après son titre professionnel n'était que chirurgien praticien ait pu révolutionné ainsi la médecine? Comment pouvait-il avoir une telle maîtrise de la physiologie et de l'anatomie comparée? Comment pouvait-il mener de front la médecine et la chirurgie? Comme nous allons le voir, c'est la formation acquise dans l'école de son frère, W. Hunter (1718-1783), qui peut rendre compte, en partie, de ce paradoxe. En effet, comme nous le verrons, J. Hunter s'est formé dans cette école à la physiologie et à la pathologie médicale autant qu'à l'anatomie et à la chirurgie. Il y avait de plus à Londres depuis le début du $18^{\mathrm{e}}$ siècle, une tradition locale d'enseignement par certains anatomistes et médecins, qui avaient une orientation anatomo-localiste, de cours privés d'anatomie auxquels était intégrée une approche anatomo-pathologique de la maladie. Ainsi depuis 1727, F. Nichols enseignait un cours d'anatomie humaine et comparée dans lequel étaient expliqués, à partir de la structure des parties, l'économie animale, la pathologie de la plupart des maladies et l'action de la plupart des médicaments ${ }^{15}$. Le $\mathrm{D}^{\mathrm{r}}$ James Douglas donnait depuis 1706 un cours d'anatomie et d'anatomie comparée. Il avait une orientation anatomo-localiste et anatomo-chirurgicale ${ }^{16}$. William Hunter a été à 
Londres l'assistant du $\mathrm{D}^{\mathrm{r}}$ Douglas et l'élève du $\mathrm{D}^{\mathrm{r}}$ Nichols et il a pu transmettre cette tradition à son frère John. De plus, William Hunter avait luimême une double formation médicale et chirurgicale. Cette double formation, Hunter avait commencé à l'acquérir auprès de W. Cullen. Cullen, il faut le souligner, avait fait un apprentissage de chirurgien-apothicaire avant de faire ses études en médecine et d'obtenir plus tard son diplôme de médecine (en 1740). Il pratiquait comme chirurgien-apothicaire dans la ville de Hamilton près de Glasgow, quand W. Hunter devint son apprenti en 1737. A l'époque, Cullen avait déjà suivi de 1735 à 1737 les cours de la Faculté de médecine d'Edimbourg. W. Hunter eut donc l'avantage d'être pendant trois ans (1737-1739) l'apprenti d'un homme de l'art qui était formé comme chirurgien et comme médecin, qui avait une expérience de «general practitioner» et qui allait devenir un des plus importants cliniciens britanniques du $18^{\mathrm{e}}$ siècle. Hunter suivit ensuite pendant un an les leçons des professeurs à la Faculté de médecine d'Edimbourg et notamment celles de Monro (primus) en anatomie et en chirurgie. Il fut ensuite élève à Londres pendant un an environ de W. Smellie, qui, en tant qu'accoucheur, se situe à la charnière de la médecine et de la chirurgie. Il devint ensuite l'élève du $\mathrm{D}^{\mathrm{r}} \mathrm{J}$. Douglas en tant qu'assistant pour les travaux de dissection et pour la chirurgie. Mais il était l'élève d'un médecin et non d'un chirurgien. Douglas donnait un des plus importants cours privé d'anatomie de l'époque à Londres et il avait une collection très importante de préparations anatomiques. Le $\mathrm{D}^{\mathrm{r}}$ Douglas était en même temps un obstétricien et il pratiquait aussi la chirurgie. Hunter a donc été en apprentissage chez un anatomiste qui personnifie une certaine inséparabilité de la médecine et de la chirurgie. Hunter a été ensuite (1741-1742) élève en chirurgie à l'hôpital Saint-George sous la direction de James Wilkie. Mais il compléta à nouveau l'aspect chirurgical de sa formation par un aspect médical en devenant au même moment élève perpétuel du $\mathrm{D}^{\mathrm{r}} \mathrm{F}$. Nichols, célèbre à Londres comme professeur d'anatomie, de médecine, d'obstétrique et de chirurgie (un peu le même profil que Douglas). W. Hunter compléta ensuite cette formation à Paris en suivant des cours d'anatomie et de chirurgie avec le médecin Ferrein et le chirurgien Le Dran. Cette double formation fut sanctionnée par le fait qu'en 1747, Hunter devint membre de la Company of Surgeons et qu'en 1750, il fut reçu docteur de l'Université de Glasgow. Nommé chirurgien-accoucheur au Lying-in Hospital en 1749 (après avoir occupé le même poste en 1748 au Middlesex Hospital), on retrouve en 1755 , Hunter avec le titre de médecin dans ce même hôpital. A partir de 1756, W. Hunter se consacre à la pratique médicale, mais 
une bonne part de sa pratique est dans le domaine de l'obstétrique qui est proche de la chirurgie. En 1756, sa nomination comme membre (licentiate) du Royal College of Physicians le contraint à abandonner son titre de chirurgien. Depuis 1754, il était, cependant très actif dans le milieu des médecins puisqu'il était membre de la Society of Hospital Physicians, qui, à partir de 1756 publia les Medical Observations and Inquiries dans lesquels il fit paraître plusieurs de ses travaux en médecine. Sa nomination en 1764 comme médecin extraordinaire de la reine Charlotte va consacrer son appartenance à la communauté médicale. Hunter était médecin obstétricien, et comme ses maîtres Smellie, Douglas et Nichols, il se situait dans une discipline où il est difficile de séparer la médecine de la chirurgie. Hunter a donc appartenu tour à tour à la profession chirurgicale et à la profession médicale. Même après avoir renoncé à son titre de chirurgien, W. Hunter a continué à enseigner, jusqu'à sa mort en 1783, la chirurgie et les opérations chirurgicales, en même temps que l'anatomie, l'obstétrique et la pathologie dans son école de la Windmill Street ${ }^{17}$. Un Bichat, plus tard, aura le même profil scientifique que W. Hunter. Formé à la chirurgie, il s'orientera par la suite vers l'anatomie et la médecine. W. Hunter était donc en position de transmettre à ses élèves, une double formation médicale et chirurgicale analogue à celle qui sera acquise plus tard par les fondateurs de l'école de Paris comme Bichat, Corvisart, Laennec, ou encore comme Broussais. Ces derniers avaient tous fait l'apprentissage de la chirurgie avant d'exercer la médecine. Cette inséparabilité de la médecine et de la chirurgie que l'on trouvait chez William Hunter allait donc nécessairement se transmettre à son frère John, comme à de nombreux autres élèves.

John Hunter travailla comme élève assistant de son frère pendant douze ans, soit de 1748 à 1760 . Simultanément, J. Hunter compléta sa formation à l'extérieur de cette école. En 1749 et 1750, il suivit la pratique chirurgicale de Cheselden à l'hôpital Chelsea. En 1751, il devint élève-chirurgien à l'hôpital Saint-Bartholomew's sous Percivall Pott. En 1754, il devint élève-chirurgien à l'hôpital Saint-George. En 1768, il deviendra membre de la Company of Surgeons et la même année il sera nommé chirurgien à l'hôpital SaintGeorge. En plus de faire un apprentissage auprès des plus grands chirurgiens cliniciens des hôpitaux de Londres, Hunter avait pu acquérir dans l'école de son frère William une formation plus générale dans toutes les branches de la médecine et il avait été associé aux recherches de ce dernier dans tous les domaines: anatomie, physiologie, pathologie médicale, obstétrique, chirurgie. J. Hunter a donc occupé une position privilégiée qui lui permettait de 
compléter l'une par l'autre la médecine et la chirurgie. En tant qu'assistant de son frère, comme prosecteur et comme démonstrateur, J. Hunter a contribué de manière importante à former les nombreux élèves qui ont fréquenté cette école de 1750 à 1760 . Pendant les 12 ans (de 1748 à 1760) où il a travaillé dans cette école, il a fait un grand nombre de dissections et de préparations anatomiques et physiologiques, ainsi qu'anatomo-pathologiques qui servaient à la recherche et à l'enseignement dans cette institution et qui ont enrichi le musée de William Hunter. Rappelons à ce propos que Math. Baillie a largement utilisé les préparations de ce musée pour composer son fameux traité d'anatomie pathologique et que c'est donc sur la base des travaux en pathologie médicale de William, mais aussi de J. Hunter que cela a été fait. De 1755 à $1760, J$. Hunter a été l'associé de son frère pour donner une partie du cours de ce dernier ou pour le remplacer quand il s'absentait ${ }^{18}$. Pendant cette période, l'école de W. Hunter était donc en réalité celle des frères Hunters.

Avec les débuts du cours de William Hunter en 1746, on assiste au commencement de l'enseignement moderne de l'anatomie en Angleterre ou de ce que Hunter annonçait comme l'art de la dissection à la manière de Paris. La dissection du corps est pratiquée par l'étudiant lui-même, chaque étudiant pouvant disposer d'un cadavre. Avant lui, même les écoles les plus célèbres en Angleterre utilisaient au plus deux cadavres pour illustrer leur cours. Et l'on faisait, le plus souvent, la démonstration des opérations chirurgicales sur le corps des animaux. L'étudiant ne disséquait jamais luimême, et il n'était qu'un spectateur passif ${ }^{19}$. Avec cette révolution de la méthode de l'enseignement, la pratique de la dissection s'accrut si rapidement qu'en 1798 il y avait trois cents étudiants d'anatomie à Londres et qu'en 1823, ils étaient mille ${ }^{20}$. On a souvent considéré l'école de W. Hunter comme étant une école d'anatomie, mais, en fait, il se donnait dans cette école un enseignement plus large que celui de l'anatomie et de la chirurgie. La description du cours donnée dans le syllabus de l'année $1782^{21}$ montre qu'il ne s'agit pas d'un enseignement limité à l'anatomie et à la chirurgie, mais d'un enseignement global qui comprend la physiologie, la pathologie (surtout l'anatomie pathologique et l'exposé des cas cliniques) et l'obstétrique ${ }^{22}$. Cette école d'anatomie embrasse pratiquement tout le domaine de la médecine. Comme le note Teacher, le cours comprend: "the whole medical curriculum with the exception of chemistry, materia medica and experimental philosophy". ${ }^{23}$ Le syllabus offre aux étudiants d'apprendre "all the arts of examining diseases". ${ }^{24} \mathrm{Il}$ est toujours question d'examiner les maladies en 
général et non pas les maladies chirurgicales uniquement ${ }^{25}$. Un des principaux avantages de l'école, selon le syllabus, c'est qu'en plus d'être une école d'anatomie, elle est une véritable école de pathologie, grâce à la quantité de préparations pathologiques qui s'y trouvent ${ }^{26}$. Il s'agit ici aussi bien de pathologie médicale que de pathologie chirurgicale. La preuve en est que M. Baillie a fondé en grande partie sur cette collection de préparations un traité d'anatomie pathologique qui est un traité de pathologie et de clinique médicales et non un traité de pathologie chirurgicale. On peut noter ici que l'école de J. Hunter, qu'il ouvrit à partir de 1770 à la Jermyn Street ${ }^{27}$, présentait les mêmes avantages que celle de son frère, à savoir que les préparations dont il disposait en faisait une école de pathologie autant que d'anatomie ou de chirurgie et, en outre, la plupart des préparations anatomo-pathologiques correspondaient à des histoires cliniques recueillies par Hunter lui-même à l'hôpital, ce qui lui permettait de mettre encore mieux en évidence les corrélations anatomo-cliniques: le syllabus du cours de J.Hunter pour l'année 1782, publié dans l'European Magazine insiste, d'ailleurs, explicitement sur ce point ${ }^{28}$. Il faut souligner le fait que Hunter déclare lui-même qu'en 1770, il a commencé à donner «des cours sur les principes de la maladie» en général et non pas seulement sur les maladies chirurgicales ${ }^{29}$.

Selon William Hunter les leçons d'anatomie devaient servir de fondation solide pour les deux arts importants de la médecine et de la chirurgie et non seulement pour la chirurgie. D'ailleurs dans les lectures d'introduction à son cours d'anatomie, W. Hunter met en avant un modèle anatomique et médical de la connaissance des maladies par l'examen anatomo-clinique ${ }^{30}$. D'autre part, la formation dispensée dans son école était considérée comme la «finishing part of education». ${ }^{31} \mathrm{Il}$ était entendu donc que les étudiants avaient déjà acquis des connaissances par l'apprentissage et/ou à l'hôpital et/ou en suivant d'autres écoles (universitaires ou non). Des écoles médicales privées comme celle de W. Hunter, ou plus tard celle de J. Hunter existaient donc en dehors des hôpitaux tout en fonctionnant en connexion étroite avec eux. Ces écoles constituaient, à côté des hôpitaux (mais le plus souvent en liaison avec eux), des centres pour l'examen des maladies et des lésions. La formation clinique médico-chirurgicale acquise par les élèves dans les écoles privées des Hunters et des huntériens (écoles privées d'«anatomie», leçons données dans les hôpitaux) était le plus souvent combinée avec une expérience pratique obtenue dans les grands hôpitaux de Londres. Ces élèves pouvaient donc profiter à la fois de l'hôpital et de l'école d'anatomie pour 
acquérir des connaissances de type anatomo-pathologique et anatomoclinique $^{31 \text { a }}$. Par exemple, un étudiant comme W. Shippen suivit pendant deux ans à Londres l'école de W. et J. Hunter tout en fréquentant assidûment les hôpitaux (Saint-George, St-Bartholomew), en tant qu'élève suivant à la fois la pratique clinique des médecins et celle des chirurgiens. Il y avait une forme d'accord, par exemple, entre W. Hunter et les chirurgiens de l'hôpital Saint-George. Les chirurgiens de cet hôpital, parmi lesquels se trouvait J.Hunter, recommandaient à leurs élèves de fréquenter l'école d'anatomie de W. Hunter et, réciproquement, W. Hunter recommandait à ses élèves de fréquenter l'hôpital Saint-George.

Une école comme celle de W. Hunter où l'on apprenait à observer les effets anatomo-pathologiques d'un grand nombre de maladies et où l'on faisait un grand nombre de dissections ne pouvait que favoriser l'interaction de la médecine et de la chirurgie et, donc, un point de vue localiste, anatomique ou «chirurgical» en médecine ou dans l'approche de la maladie. D'autre part, le syllabus du cours de 1782 insiste sur la valeur d'une approche «quantitative» des faits pathologiques: l'école permet l'observation d'un grand nombre de maladies, la pratique d'un grand nombre de dissections et d'opérations de chirurgie, et aussi, l'utilisation et la fabrication d'un grand nombre de préparations pathologiques illustrant les maladies ${ }^{32}$. Dans une telle école, on cultive donc non seulement l'anatomie et la chirurgie, mais aussi la physiologie et la médecine. On a donc, une forme d'unification de facto entre les différentes branches de l'art médical. Etant donné que cette école a fonctionné pendant de nombreuses années (le syllabus du cours de 1782 rappelle que cette école existe depuis trente-six ans) on y a formé un très grand nombre d'élèves. A l'ouverture de son école en 1746, W. Hunter n'avait que dix ou quinze étudiants, mais en 1756, il y avait déjà une centaine d'élèves à son cours et il donnait ce cours deux fois par an ${ }^{33}$. Hunter avait donc environ deux cents élèves par an à son cours. De 1756 à 1783, il aurait donc eu environ plus de 5000 élèves (si nous ne comptons qu'à partir de 1756). Si Hunter n'a eu, en réalité, que 100 élèves par an, cela fait tout de même près de trois milles élèves en vingt-sept ans. Il est vrai que tous les élèves qui suivaient le cours ne travaillaient pas forcément tous dans la salle de dissection. Cependant un nombre important d'étudiants disséquaient, ce qui représente une proportion considérable sur une telle quantité d'élèves. Pour dix guinées on devenait un élève perpétuel aux leçons d'anatomie et on pouvait y assister aussi longtemps que l'on voulait. Tout élève perpétuel aux leçons d'anatomie, pouvait pour dix guinées de plus devenir un élève 
perpétuel de l'école de dissection. Les élèves perpétuels à la salle de dissection pouvaient voir toutes les préparations faites pour les leçons et ils pouvaient disséquer et faire des injections à loisir, à condition de payer pour le sujet qu'ils utilisaient. Ceux qui ne voulaient disséquer que pour une courte période pouvaient disséquer un corps en payant deux guinées pour un étudiant ou une guinée pour deux étudiants ${ }^{34}$. Malgré les difficultés qu'il y avait à se procurer des sujets pour la dissection dans les écoles privées d'anatomie à Londres au $18^{\mathrm{e}}$ siècle, William Hunter était en mesure de se procurer un matériel suffisant pour ses recherches et pour la formation des élèves. Tout comme son frère John et d'autres enseignants des écoles privées par la suite, W. Hunter obtenait des cadavres pour l'autopsie par l'entremise d'un réseau bien établi d'exhumateurs de sépultures et de vendeurs de cadavres, puisqu'on ne pouvait pas les obtenir par des voies légales ${ }^{35}$. En tout cas, certains témoignages comme ceux du médecin Fordyce, qui nous apprend que lorsqu'il était élève de Hunter, il avait eu trois cadavres à sa disposition pour s'exercer à la dissection, nous montrent qu'il y avait un matériel suffisant pour le cours d'anatomie pratique ${ }^{36}$. Par ailleurs, le nombre de préparations anatomiques et pathologiques du musée de W. Hunter à Glasgow s'élève à 2607 et il est certain que pour constituer une telle collection, W. Hunter a dû disposer d'un matériel anatomique très important ${ }^{36}$.

Comme W. Hunter, John Hunter a formé plusieurs centaines d'élèves ${ }^{37}$. Or ces générations d'élèves ont été formés, comme ceux de W. Hunter, dans une problématique d'interpénétration de la médecine et de la chirurgie qui a été exposée très explicitement par l'élève de J.Hunter, J.Abernethy (1764-1831) ${ }^{38}$. C'est en 1770 , que Hunter a ouvert sa propre école privée à la Jermyn Street. Il y a une différence entre l'école de W. Hunter et celle de J.Hunter qui est la suivante. Quand on parle des élèves de W. Hunter, on parle de ceux qui ont suivi ses cours et qui ont travaillé dans les salles de dissection de son école d'anatomie de la Windmill Street (à partir de 1767) ou d'ailleurs auparavant. John Hunter, par contre, a eu surtout des élèves à l'hôpital auxquels il donnait une instruction clinique et, de plus, il a eu des élèves qui ont suivi son enseignement privé et dont certains ont travaillé dans sa salle de dissection. Les élèves hospitaliers de Hunter et ceux qui suivaient son cours privé pouvaient être les mêmes. A partir de 1768, Hunter a commencé à avoir des élèves à l'hôpital Saint-George qu'il instruisait dans la pratique clinique. De 1768 à 1793 , il a eu ainsi plusieurs centaines d'élèves à l'hôpital. Les élèves de son école privée, où il donnait un enseignement 
théorique, étaient moins nombreux. Au début, ses élèves de l'hôpital étaient admis gratuitement à son cours. En 1783, J. Hunter installa son école, son musée et sa salle de dissection dans ses deux maisons de Leicester Fields et de Castle Street et il y demeura jusqu'à sa mort en 1793. Ce qui est certain, c'est que, à l'hôpital ou dans son école privée, et pour un certain nombre d'étudiants dans les deux simultanément, J. Hunter a pu former un grand nombre d'étudiants dans cet esprit d'inséparabilité de la médecine et de la chirurgie. M. Baillie est un des meilleurs exemples de cette double formation, à la fois en médecine et en chirurgie. De 1780 à 1783, M. Baillie, a suivi les leçons d'anatomie et les travaux de dissection dans l'école de W. Hunter ainsi que les cours du $\mathrm{D}^{\mathrm{r}} \mathrm{G}$. Fordyce (1736-1802) sur la pratique de la médecine, la matière médicale et la chimie. En même temps, Baillie suivait les leçons de John Hunter sur la chirurgie ainsi que celles des $\mathrm{D}^{\mathrm{r}}$ Denman et Osborne sur l'obstétrique ${ }^{39}$. En 1783, après la mort de William Hunter, M. Baillie devint un élève perpétuel (perpetual pupil) en médecine à l'hôpital Saint-George ${ }^{40}$ et il devint, en même temps, pendant plus d'un an, élève en chirurgie à l'hôpital (auprès de J.Hunter) ${ }^{41}$. Comme l'a souligné Baillie lui-même, c'était la conviction qu'il y avait l'unité et l'interdépendance les plus étroites entre la médecine et la chirurgie qui l'avait poussé à faire cette double formation ${ }^{42}$.

En 1787, Baillie est nommé médecin à l'hôpital Saint-George. En 1789, il obtient son titre de docteur en médecine de l'Université d'Oxford et la même année, il est nommé fellow du Royal College of Physicians of London. Baillie est demeuré comme médecin à l'hôpital Saint-George jusqu'en $1799^{43}$. La même année, Baillie devait abandonner son enseignement à l'école de la Windmill Street où il avait succédé à W. Hunter. Baillie a résumé ainsi son expérience de médecin hospitalier: "During my situation there (SaintGeorge), I was not only attentive as I could be to the cases of my patients but embraced every opportunity of examining the morbid appearances after death". ${ }^{44}$ En tant que médecin praticien à l'hôpital, Baillie accordait donc un intérêt considérable à la pathologie. Baillie avait commencé à enseigner dans l'école de la Windmill Street en 1782. Après la mort de William Hunter (1783), il devint un professeur à part entière (full lecturer) et il le demeura pendant 16 ans $^{45}$. Il était le partenaire de W. Cruikshank, qui avait été le collaborateur de William Hunter à l'école de la Windmill Street depuis 1771. Cruikshank avait également une formation à la fois médicale et chirurgicale. Le succès de Baillie comme enseignant fut tel que le nombre des élèves de la Windmill Street ne diminua pas après la mort de son fondateur ${ }^{46}$. Pendant 
toutes ces années, il y avait donc à la Windmill School des générations d'élèves qui pouvaient suivre un enseignement donné par un professeur qui était formé à la fois en médecine et en chirurgie, qui intégrait la médecine et la chirurgie dans une approche anatomique et localiste ou anatomo-clinique de la maladie, et qui possédait une expérience clinique hospitalière et une expérience d'anatomo-pathologiste de première importance. Baillie avait autant d'élèves au moins que William Hunter. En 16 ans, il a donc pu former sur une telle base anatomo-clinique entre 1500 et 3000 élèves (sinon plus), ce qui est considérable. Wardrop a souligné que cette volonté de Baillie d'unifier et d'intégrer les connaissances médicales et chirurgicales et de donner une telle base à la formation médicale, s'expliquait d'abord chez lui par l'exemple donné par ses oncles ${ }^{47}$.

Il convient de revenir maintenant un peu en arrière pour se demander quel était le profil des élèves qui suivaient l'école de W. Hunter. Les cours de Hunter étaient suivis aussi bien par des élèves qui se destinaient à la médecine ou à l'obstétrique que par des élèves qui se destinaient à la chirurgie. Mais, en plus, parmi ceux qui fréquentaient cette école, il y en avait qui étaient déjà diplômés en médecine ou dans une autre discipline. Les élèves américains comme Morgan, Rush, Kuhn, Shippen et Physick ont tous obtenu plus tard un diplôme de médecine. Ils suivirent les cours de W. Hunter et l'école de dissection à l'époque où J. Hunter était l'associé de son frère ${ }^{48}$. Shippen, par exemple, passa deux ans à Londres où il suvit l'école de Hunter et où il fréquenta les hôpitaux. Un an plus tard, en 1761, il obtint son titre de docteur en médecine de l'Université d'Edimbourg ${ }^{49}$.

Un nombre important d'élèves de William (et plus tard de J.Hunter) se caractérisent par le fait qu'ils ont une formation à la fois en médecine et en chirurgie, même s'ils n'ont pas pour autant les deux diplômes. Les médecins qui suivaient l'école de William Hunter se formaient à la chirurgie et inversement, les chirurgiens qui suivaient ce cours acquéraient une formation en médecine. Nous ne pouvons donner ici que quelques exemples. Ainsi W.Stark avait déjà acquis une formation médicale à Edimbourg qu'il compléta à partir de 1765 par une formation dans l'école de William Hunter où il put acquérir des connaissances chirurgicales. Il entra la même année comme élève à l'hôpital Saint-George ${ }^{50}$. G. Fordyce, avait déjà obtenu son diplôme de médecin de l'Université d'Edimbourg en 1758, avant de suivre l'école de W. Hunter, où un médecin devait nécessairement acquérir une initiation à la chirurgie ${ }^{51}$. W. Hewson a acquis dans l'école de W. Hunter une formation médicale autant que chirurgicale et il s'est distingué par des 
travaux de physiologie et d'anatomie comparée sur le sang et le système lymphatique. Après avoir succédé comme prosecteur à J. Hunter et passé un an à étudier à la Faculté de Médecine de l'Université d'Edimbourg, Hewson devint en $1764^{52}$ le partenaire de l'école de W. Hunter. Les collaborateurs directs de Hunter étaient considérés comme des anatomistes plutôt que comme des chirurgiens. Après Hewson, c'est le cas de W. Cruikshank. Cruikshank avait aussi une formation à la fois médicale et chirurgicale. Avant de devenir l'élève puis le partenaire de W. Hunter en 1772, Cruikshank avait été élève en médecine du $\mathrm{D}^{\mathrm{r}}$ John Moore ${ }^{53}$. Avec W. Hunter, puis avec Baillie, Cruikshank enseigna l'anatomie, la pathologie, la chirurgie, la physiologie et l'obstétrique. Il eut une pratique considérable de chirurgien. Il reçut un diplôme honoraire de médecin de l'Université de Glasgow en 1797. Comme on l'a vu, M. Baillie avait une double formation médicale et chirurgicale. E. Jenner s'était formé à la chirurgie auprès de John Hunter et il avait obtenu un diplôme de médecin en 1792 de l'Université de St. Andrew's ${ }^{54}$. Dans la génération suivante des huntériens, on voit que les chirurgiens comme A. Cooper ou B. Brodie se sont formés en suivant aussi des cours de médecine. A. Cooper a suivi des cours de médecine à Edimbourg ${ }^{55}$ et B. Brodie les cours de Chrichton à Londres ${ }^{56}$. W. Babington (1756-1833) était membre depuis $1787 \mathrm{du}$ Lyceum Medicum Londinense ainsi que de la Guy's Hospital Physical Society, deux sociétés médicales où Hunter exerçait une influence prépondérante. Babington devint un des principaux professeurs de médecine à Londres à la fin du $18^{\mathrm{e}}$ siècle. Il avait été formé d'abord comme chirurgien-apothicaire, avait étudié à l'hôpital Guy's et avait été chirurgien à l'hôpital Haslar, puis apothicaire à l'hôpital Guy's où il fut nommé médecin quand il obtint son diplôme de docteur de l'université d'Aberdeen en $1795^{57}$. Autre exemple donc de formation à la fois médicale et chirurgicale. Richard Bright (1789-1858), diplômé en médecine d'Edimbourg en 1812 avait aussi acquis une formation en chirurgie et en médecine avec les huntériens A. Cooper, les deux Cline, Travers et Babington. C'est Cooper notamment qui orienta Bright vers l'étude anatomo-pathologique des maladies ${ }^{58}$. Un des effets de cette double formation des huntériens, c'est que certains d'entre eux, comme Philip Syng Physic (1768-1837) ont été reconnus par la suite aussi bien comme médecin que comme chirurgien ${ }^{59}$. Souvent, les huntériens ont produit des travaux à la fois en médecine et en chirurgie, ou qui étaient pertinents pour les deux branches de l'art. Il en va ainsi du traité sur l'inflammation de J. Thomson, qui était professeur de chirurgie militaire à l'université d'Edimbourg. Ce livre était en fait un traité 
de pathologie médicale et il était aussi utile au chirurgien qu'au médecin ${ }^{60}$. De même Travers, autre huntérien, publia des travaux sur la physiologie de l'inflammation qui relevaient largement de la médecine. On disait de lui qu'il était un très bon chirurgien, mais qu'il aurait fait un meilleur médecin encore $^{61}$. On peut noter ici que le rapprochement et l'interaction de la médecine et de la chirurgie s'est aussi opéré pour les huntériens à travers certaines sociétés pour l'avancement de la médecine et de la chirurgie, à la création desquelles J. Hunter a pris une part déterminante. On pourrait dire même que ces sociétés faisaient partie du réseau d'influence de l'école huntérienne. Dès 1783, pour promouvoir l'interaction fructueuse entre la médecine et la chirurgie, J. Hunter et G. Fordyce, médecin à l'hôpital SaintGeorge, avaient fondé ensemble une société pour promouvoir les conaissances médico-chirurgicales (Society for the Improvement of Medical and Surgical Knowledge) qui publiera les Transactions for the Improvement of Medical and Surgical Knowledge. En 1785, J.Hunter créa aussi, toujours avec Fordyce, pour développer les connaissances médico-chirurgicales, une société appelée le Lyceum medicum Londinense, ouverte aux médecins et aux chirurgiens et aux étudiants en médecine comme aux étudiants en chirurgie. Les enseignements de Hunter exerçaient une grande influence aussi au niveau de la Guy's Hospital Physical Society (1771), qui accueillait les travaux des médecins aussi bien que ceux des chirurgiens. Un grand nombre d'élèves de Hunter étaient membres de cette société. On peut indiquer aussi que, à l'instigation souvent des huntériens, ce genre de sociétés médicochirurgicales se sont multipliées à Londres à la fin du $18^{\mathrm{e}}$ et au début du $19^{\mathrm{e}}$ siècles et que leurs publications où l'on trouve à la fois les travaux des médecins et des chirurgiens ou des chirurgiens-apothicaires attestent le fait que l'on avait une problématique unificatrice pour ce qui est des rapports entre médecine et chirurgie et qu'un travail de recherche se faisait en commun et en interaction ${ }^{62}$.

Le fait que, dans l'école huntérienne, on ait pu cultiver et enseigner simultanément l'anatomie, la chirurgie, la physiologie, la médecine, l'anatomie pathologique, etc. a donc eu pour effet de rendre possible une forme développée d'interaction et d'intégration entre ces différents domaines. Cette interaction a permis à son tour que se systématise dans cette école un point de vue chirurgical (anatomique, localiste) en médecine clinique, et, réciproquement, un point de vue physiologique et pathologique en chirurgie. C'est ce qui explique, sans doute, que l'on ait vu sortir de cette école des figures commes celles de M. Baillie. Son traité d'anatomie pathologique est 
une production typique de l'école huntérienne. Comme déjà les travaux des Hunters sur les corrélations anatomo-cliniques et la pathologie des organes et tissus, ce traité montre à quel point la clinique avait intégré, dans cette école, l'approche anatomique et localiste de la chirurgie. L'école de William et John Hunter et de leurs collaborateurs comme M. Baillie avait done déjà commencé à réaliser largement au cours de la seconde moitié du $18^{\mathrm{e}}$ siècle un programme qui allait devenir celui de l'Ecole de Paris, à savoir, importer dans le domaine de la médecine, la problématique anatomique et localisatrice de la chirurgie. Ce programme ne sera réalisé entièrement qu'à partir des années 1820 avec Laennec et des cliniciens comme Louis, mais, auparavant, l'école des Hunters avait déjà montré la voie et s'était engagée assez avant dans cette carrière. Les médecins de l'Ecole de Paris, comme Dezeimeris et bien d'autres, ont d'ailleurs explicitement reconnu que les œuvres des Hunters et de leurs élèves avaient fonctionné comme un paradigme essentiel dans le développement de la problématique clinique médicale et chirurgicale de la médecine parisienne. Bien avant l'Ecole Clinique de Paris, l'école huntérienne a donc fait fonctionner, sur le plan scientifique, une forme d'unification entre médecine et chirurgie, nonobstant le fait que, sur le plan social et professionnel, il subsistait, en Angleterre, une séparation et une inégalité entre les deux branches de l'art de guérir ${ }^{63}$.

\section{Notes}

1 Cf. par exemple, Imbault-Huart, M.J., «Les chirurgiens et l'esprit chirurgical en France au XVIII ${ }^{e}$ siècle», Clio Medica, 15 no 3/4 (1981) pp.143-157. Pour une bibliographie complète sur ce point, voir Keel, $O$. "The Politics of health and the institutionalisation of clinical practices in Europe in the second half of the eighteenth century" in Bynum, W. F. \& Porter, R. (eds) William Hunter and the eighteenth-century medical world, Cambridge University Press, 1985, 207-256; voir p. 208, note 1 .

2 Imbault-Huart, ibid.

3 Cf. Temkin, 0 . "The role of surgery in the rise of modern medical thought", Bull. Hist. Med. 25 (1951): 248-259.

4 Temkin, ibid.; Cf. aussi Keel, op. cit. (n.1).

5 Voir aussi sur ce point Keel, op. cit. (n.1) et id. «La place et la fonction des modèles étrangers dans la constitution de la problématique hospitalière de l'Ecole de Paris», History and Philosophy of the Life Sciences, 6 (1984), 41-73. Voir aussi Reiser, S.J., Medicine and the reign of technology, Cambridge University Press, 1978, p. 19.

6 Temkin, op.cit., (n.3), pp. 257-258.

7 Voir plus loin.

8 Temkin, ibid., p. 248. 
9 Cité par Teacher, J.H., Catalogue of the Anatomical and Pathological Preparations in the Hunterian Museum, University of Glasgow, 2 vols (Glasgow, 1900), p.22.

10 Cf. Keel, O. La généalogie de l'histopathologie, Paris, 1979. Id. «La patholgoie tissulaire de J. Hunter», Gesnerus 37 (1980), 1/2: 47-61, id. «J. Hunter at X. Bichat: les rapports de leurs travaux en pathologie tissulaire». Actes du XXVII Congrès International d'histoire de la Médecine, Barcelone, 1981, II, pp.535-549; id. «Les conditions de la décomposition analytique de l'organisme: Haller, Hunter, Bichat» in Les Idéologues; numéro spécial de Etudes philosophiques $\mathrm{n}^{\circ} 1$ (1982); 37-62; id. op. cit. (n. 5), (1984), p. 70 sq. Voir aussi Klemperer P., "John Hunter's contribution to pathology", Bull. NY Acad. Med. 36, 281-284.

11 Voir Cross, S. J.: "John Hunter, the Animal Economy, and Late Eighteenth-century Physiological Discourse", Studies in the History of Biology, V (1981), p. 309 sq.

12 Cf. par exemple Gloyne, S. R.: John Hunter, Edimbourg, 1950, p. V. Cf. aussi Gross, S. D., John Hunter and his pupils, Philadelphia, 1881, p. 68.

13 Mather G.E.R., Two Great Scotsmen: the brothers William and John Hunter. Glasgow 1893 , p. 183 sq. Voir aussi Jacyna, L.S. "Images of John Hunter in the nineteenth century", Hist. Sci., XXI (1983), 87-108 qui établit une liste d'une série d'évaluations analogues de l'œuvre de J.Hunter au $19^{\mathrm{e}}$ siècle. Sur la physiologie et l'anatomie comparées chez Hunter, voir aussi, inter alia, Ritterbush P. D., Overtures to biology: The speculations of eighteenth-century naturalist, New-Haven, London, 1964; Duchesneau, F., "Vitalism in late eighteenth century physiology: the cases of Barthez, Blumenbach and John Hunter" in Bynum, W. F. \& Porter, R. (eds), op. cit. (n. 1), 259-296; Rolfe W. D.I. "William and John Hunter: breaking the Great Chain of Being", ibid., 297-322. Cross, op. cit.

14 Lectures on Physiology, Zoology and the Natural History of Man, Londres 1819, p.64. Cité in Cross, op. cit., p. 94 (n. 114).

15 Cf. Peachey G. C.: A memoir of William and John Hunter. Plymouth, 1924; p. 15. Peachey donne une description des différents cours privés d'anatomie qui se sont donnés Londres au $18^{\mathrm{e}}$ siècle avant celui de William Hunter. Cf. p. 1 sq.

16 Ibid. pp. 14-15.

17 Sur tous ces points de la biographie et de la carrière de W. Hunter voir inter alia, Hingston Fox, R.: William Hunter, Anatomist, Physician, Obstetrician, with Notices of his Friends (Londres, 1901); Teacher, op. cit. Peachey, op. cit.; Beekman, F., "William Hunter's Early Medical Education". Journal of the History of Medicine, V (1950), 172-184; Brock, C. H. (ed.), William Hunter 1718-1783. A Memoir by Samuel Foart Simmons and John Hunter, University of Glasgow Press, 1983 ainsi que Bynum W. F. \& Porter Roy, op. cit. (n. 1), notamment Porter, R., "William Hunter: a surgeon and a gentleman", p. 7-34; et Brock, C. H. "The happiness of riches", 35-56; Illingworth, Ch.: The story of William Hunter, Edimbourg, 1967.

18 Sur tous ces points de la biographie et de la carrière de J. Hunter, voir inter alia Peachey, op. cit.; Cross, op. cit.; Brock, op. cit.; Mather, op. cit.; Hingston Fox, op. cit.; Beekman F., "The self-Education of the Young John Hunter", Journal of the History of Medicine, 6 (1951): 500-515; id. "Teacher and Pupil: The Brothers William and John Hunter from 1748-1760", Bull. Hist. Med., 28 (1954): 501-514; Gloyne, op. cit.; Home E. "A short account of the Life of the author", in John Hunter, A treatise on blood, inflammation and 
gun-shot wounds, Londres, 1794; pp.XIII-LXVII; Adams, J., Memoirs of the Life and doctrines of the late John Hunter, Esq. Londres, 1818; Ottley, D. "The Life of John Hunter, F. R.S." in The Works of John Hunter, F.R.S., ed. Palmer J. Londres 1835, Paget. S. John Hunter. Londres, 1897; 1960; Dobson, J.: John Hunter Edimbourg, 1969, Quist, G. John Hunter (1728-1793), Londres, 1981. Kobler, J., The reluctant surgeon, New York, 1960.

19 Cf. Peachey; p. 80 et p. 98; Guttmacher, A. F.: "Bootlegging bodies: a history of bodysnatching", Bulletin of the Society of Medical History of Chicago, Vol. IV, no 4, 1935, p. 360 sq. Cf. aussi Report from the Select Committee on Anatomy, 1828, printed for the House of Commons, p.4. Cependant, avant W. Hunter, certains cours avaient été annoncés où les étudiants pouvaient disséquer de leurs propres mains. Cf. Peachey, ibid, p.39 et 96. Certains étudiants pouvaient disséquer aussi dans les hôpitaux avant 1746.

20 Select Committee on Anatomy, p.4. Guttmacher, ibid.

21 Ce syllabus a été publié en 1782 dans l'European Magazine. Il est cité intégralement in Peachey, op. cit., pp.128-130.

22 Teacher, op. cit., p. 22 et p. 65. Sur l'influence de cette école cf. Thomson, S. C.: "The great Windmill Street School", Bull.Hist. Med. 12 (1942): 377-391.

23 Teacher, op. cit., p. 65 et Peachey, op. cit. p. 128.

24 Peachey, op. cit., p. 130.

25 "In this school so many subjects are dissected in the course of a winter, so many diseases examined, and occasional demonstrations of all parts of the body so often repeated, that for acquiring substantiel knowledge it is reckoned preferable to every other kind of study and to be the finishing part of education". Cf. Peachey, op. cit., p. 130; souligné par nous.

26 "But above all the inestimable treasure of preparations, and especially of diseases, which the Museum Contains, and which are introduced into the Lectures, such as no teacher was ever possessed of before". Peachey, ibid; souligné par nous.

27 Cf. Peachey, op. cit., p.161. C. aussi note 18.

28 "This course of lecture is illustrated by a collection of diseases and of comparative anatomy which in point of curiosity, accuracy and comprehension is equal to any collection in the world. It has been made by Mr. Hunter himself and what chiefly contributes to its extraordinary value and advantage is, that he knows the particular history of the greater part of the diseases which he has preserved - the patients where under his observations in the hospitals - he has minuted the progress and accounted for the various appearances and effects of each disease, with a fidelity that now renders his collection a most instructive school for the students". Cité par Peachey, op. cit., p. 171. Souligné par nous,

29 Cf. Peachey, op. cit. p. 161. Cf. Palmer, J. (ed): The Works of John Hunter, Londres, 1837, vol. 3,1 .

30 "This advantage, which we receive from anatomy of finding out the real disease after death, has been generally adopted by the moderns, that the cases already published are almost innumerable: Mangetus, Morgagni, indeed many of the best modern writings in physic are full of them. And if we look among the physicians of the best character, and observer those who have the art itself, rather than the craft of the profession at heart: we shall find them constantly taking pains to procure leave to examine the bodies of the patients after death; desirous that it may be done by experienced anatomists (a circumstance often of the highest importance) and unhappy when they cannot procure this opportunity of 
improving themselves and their art. Were $I$ to guess at the most probable future improvements in physic, I should say, that they would arise from a more general and more accurate examination of disease after death. And were I to place a man of proper talents in the most direct road for becoming truly great in his profession, I would choose a good practical anatomist and put him into a large hospital to attend the sick and dissect the dead." Cf. Two introductory lectures, delivered by Sr. William Hunter, Londres, 1784, p. 72. Souligné par nous.

31 Voir note 25.

$31^{\text {a }}$ Sur ces points, cf. Keel, op. cit. (n. 1) 1985, P. 229 sq.

32 Cf. Syllabus du cours de 1782, cité par Peachey, op. cit., p. 130.

33 Cf. Paget, op. cit., p.61, Porter, op. cit., p. 22; Brock, op. cit., p. 38; Peachey, op. cit., p. 47 et p. 93 .

34. Peachey, ibid. p. 127. Ces tarifs sont ceux en vigueur à la fin de la vie de Hunter. En 1756, ils étaient assez inférieurs. Cf. Peachey, op. cit., p. 47.

35 Cf. Select Committee on Anatomy, 1828, p. 17 sq. Kobler, op. cit. p. 66; Guttmacher, op. cit.

36 Peachey, p. 67; Teacher, op. cit., p. LXVII; Porter, op. cit., p. 23.

$36^{\text {a }}$ Teacher, op. cit. Nous avons d'ailleurs le témoignage de Hunter lui-même qui montre qu'un grand nombre de dissections se pratiquaient dans son école. D'après Hunter, l'étudiant devait fréquenter la salle de dissection où "he will see the preparatory dissection for every lecture, which make the lecture itself more intelligible, and fix it deeper in the mind; he will see all the principal parts dissected over and over again; whatever he finds he does not understand there is such a nomber of bodies dissected in succession that he will at any time have the opportunity of attending to that particular object, and of getting it explained to him; he will see all the operations of surgery performed and explained again and again, and he will see the practice of all the arts of making preparations". Two introductory lectures, p. 109, cité par Teacher, op. cit., p. 68.

37 Cf. Finch, E. "The influence of the Hunters on Medical Education", Annals of the Royal College of Surgeons 20 (1957): 219, Quist, op. cit. p.25; Porritt, A. Hunterian Oration 1967. "John Hunter: distant echoes". Annals of the Royal College of Surgeons of England, 1967, vol.41, pp.8-9; Keel, op. cit., n.1 (1985), p.227 et p.229. Peachey, op. cit., p.150; Cf. Taylor T.; Leicester Square, its associations and its worthies, with a sketch of Hunter's scientific character and works by Richard Owen (Londres, 1874): "They (les étudiants) followed Hunter through the wards in crowds". Cf. aussi Gross, op. cit. (n.12).

38 «Medicine is one and indivisible, it must be learnt as a whole, for no part can be understood if learnt separately. The physician must understand surgery; the surgeon, the medical treatment of disease. Indeed, it is from the evidence afforded by external disease, that we are able to judge of the nature and progress of those which are internals. The Hunterian Oration, Londres, 1819 , p. 30. Il y a ici une analogie avec le dictum de Laennec: «En un mot, j'ai tâché de mettre sous le rapport du diagnostic les lésions organiques internes sur la même ligne que les maladies chirugicales...». Traité de l'auscultation médiate, $2^{\mathrm{e}}$ éd., Paris, 1826, vol.1, p.XXV.

39 Cf. Rodin, A.E., The influence of Mathew Baillie's Morbid Anatomy, Biography, Evaluation and Reprint, Springfield, Ill. 1973, p. 8; M.Baillie, An Autobiography. Publié in Practitioner, 1896, LVII, p. 54.

40 Baillie, An Autobiography, p. 56. 
41 Ibid.; Rodin, ibid. p. 8. Cf. aussi Keel, op. cit. (n. 1), 1985, p. 230.

42 "It appeared to me that medicine and surgery were so connected together that by knowing something of the latter I should become more satisfactorily acquainted with the former, and in this opinion after long experience, I think that I have not been mistaken". Baillie, An Autobiography, p.56.

43 Rodin, op. cit., p. 11.

44 Autobiography, p.57.

45 Rodin, ibid.

46 Cf. Wardrop, J.; Collected works of Matthew Baillie to which is prefixed an account of his life, Londres, 1825, p. XXVI.

47 "The history of his uncles, and the important advantages which they had retired from a general and comprehensive knowledge of their profession, were alone sufficient to point out to him the propriety of considering medicine as one science..." ibid, p.XXIII. (Souligné par nous.)

48 Cf. par exemple Bell, W. J. "Philadelphia Medical Students in Europe, 1750-1800" in The Colonial Physician and Other Essays (New York, 1975). Cf. aussi Corner, B.C.: William Shippen, Jr.: Pioneer in American Medical Education et idem.: The Autobiography of Benjamin Rush, Princeton, N.J., 1948, pp.52-53. Philadelphia 1951; Bell, W.J., John Morgan, Continental Doctor (Philadelphie), 1965.

49 Corner, op. cit., 1951, p. 1 sq, pp.1-49.

50 Cf. Keel, op. cit. (1979), pp.107-108 et «W.Stark» in: Hirsch, A. et Gurlt, E., Biographisches Lexikon der hervorragendsten Ärzte.

51 Cf. "Fordyce George", in Dictionary of National Biography; cf. aussi in Munk, W.: Roll of the Royal College of Physicians.

52 Cf. "Hewson, W." in Dict. Nat.Biog. Peachey, op. cit., p.118; Brock, op. cit., p.50. Hewson avait commencé par un apprentissage de chirurgien-apothicaire.

53 Cf. "Cruikshank, W." in Dict. Nat. Biog. Brock, ibid.

54 "Jenner, E." in Dict. Nat. Biog. et in Hirsch et Gurtl, op. cit.

55 "Cooper, A." in Dict. Nat. Biog.

56 Brodie, B. in Dict. Nat.Biog. Cf. aussi Hawkins Ch. (ed), The Works of Sir Benjamin Collins Brodie with an autobiography, Londres, 1865, p. 21.

57 "Babington, W." in Dict. Nat. Biogr. Cf. aussi Hirsch et Gurtl, op. cit.

58 "Bright, R." in Dict. Nat.Biogr., ef. aussi Bright, P., Dr. Richard Bright (1789-1858), Londres, 1983. p. 68 sq. et p. 73 sq.

59 Cf. Gross, S.D., John Hunter and his pupils. Philadelphie 1881: "Nor was he known simply as a surgeon, he ranked high, very high, also as a physician".p. 87.

60 Cf. Gross, ibid. p. 90.

61 Cf. Gross, ibid.p. 97.

62 Sur ces sociétés médicales et l'influence de Hunter, cf. Regulations and laws of the Lyceum Medicum Londinense held at Mr. John Hunter's Lecture-room, Castle Street, Leicester Square, Londres 1788. Cf. p. 4 sur les différentes classes de membres. Etaient admis aussi bien les médecins, les chirurgiens ou les apothicaires diplômés dans la première classe. Dans la seconde classe, il était exigé des membres d'avoir au moins "attended an hospital, one course of lectures on anatomy and the practice of physic". Cf. aussi Newton Pitt, G., "Reflection on John Hunter as a physician and on his relation to the medical societies of 
the last century", The Lancet, 1896, vol. 1, 1270-1274. Cf. aussi Wolstenholme, G., "Societies for the Improvement of Medical and chirurgical Knowledge" Hunterian Society Transactions, 1974-75-76, vol.33-34: 124-235. Cf. aussi Porritt, A., op. cit., voir note 37. Power d'A.: British medical societies, Londres, 1933; Lawrence, S. C.: "Desirous of improvement in medicine: pupils and practitioners in the medical societies at Guy's and St.-Bartholomew's Hospital, 1795-1815", Bull. Hist. Med. 59 (1985): 89-104. Selon Newton Pitt, J.Hunter influençait à travers ces sociétés médico-chirurgicales environ 500 élèves et praticiens. "The medical societies, écrit-il, were looked upon as merely part of the educational machinery of the metropolis", op. cit., p. 1274. Le musée de J. Hunter était au service des membres du Lyceum Medicum Londinense. (ibid). La liste des membres de cette société s'élève à 303 personnes en 1788 , dont 70 dans la première classe, 197 dans la seconde et 30 dans la troisième. Cf. Regulations ... of the Lyceum, pp. 27-46.

63 Sur ces points, voir Keel, La gênéalogie de l'histopathologie. Paris, 1979, p. 55 sq.; id. op. cit. (1981) voir note no 5. Voir aussi Keel, op. cit., 1985, voir note 1.; id. «La formation de la problématique de l'anatomie des systèmes selon Laennec» in Laennec 1781-1826. Revue du Palais de la Découverte, 22, 1981: 189-207; id.; «La place et la fonction des modèles étrangers dans la constitution de la problématique hospitalière de l'école de Paris». History and Philosophy of the Life Sciences 6 (1984): 41-73, spécialement p. 68 sq.

\section{Summary}

It is well known that the establishment of a closer connection between medicine and surgery which took place in the 18th and 19th centuries was a very important factor in the formation of an anatomo-clinical approach to disease. However, historians have until now focused primarily on the role played by the French medical school (especially the Paris Clinical School) in integrating medicine and surgery at the beginning of the 19th century.

This paper demonstrates that the English school of William and John Hunter (and their followers) was also instrumental in bringing about the integration of medicine and surgery, leading thus to an anatomical and localist concept of disease. The hunterian schools, in connection with the London hospitals, provided training which offered the opportunity to practice and learn, in the same institutions, anatomy, surgery, physiology, medicine and pathological anatomy. Many of the pupils had already been trained in medicine or surgery. This brought about the systematic interaction and eventual integration of these various fields.

This paper concludes that the hunterian tradition integrated medicine and surgery, at conceptual and clinical levels, even tough these fields were, at that time in England, still separated at social and professional levels. These developments began in London in the second half of the 18th century before a similar process was set in motion by the Paris Clinical School. This process of conceptual and clinical integration of medicine and surgery was developed by the hunterians, in parallel with the Paris School, well into the 19th century.

Othmar Keel, Professeur, Département d'Histoire, Université de Montréal

C. P. 6128, Succ. A, Montréal, P.Q., Canada H3C 3J7 